\title{
UM MÉTODO HEURÍSTICO BASEADO EM PROGRAMAÇÃO DINÂMICA PARA O PROBLEMA DE CORTE BIDIMENSIONAL \\ GUILHOTINADO RESTRITO
}

GESTÃO $\&$ PRODUÇÃO

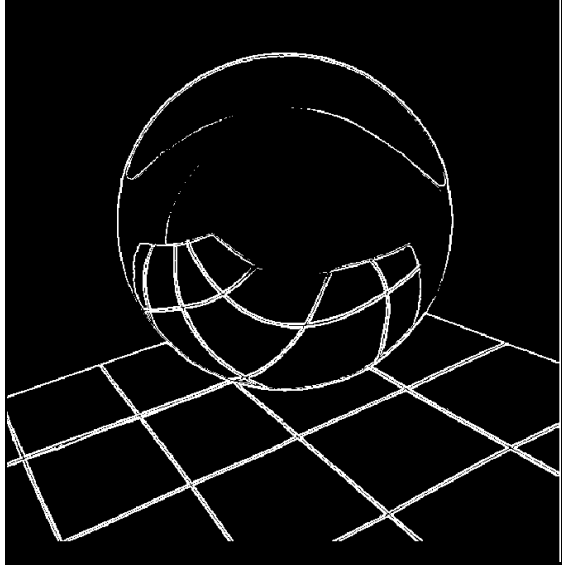

v.9, n.1, p.78-92, abr. 2002

\section{Rejane Joas Silveira \\ Reinaldo Morabito}

Departamento de Engenharia de Produção

Universidade Federal de São Carlos

São Carlos - SP

E-mails: rejane_silveira@bol.com.br

morabito@power.ufscar.br

\section{Resumo}

Neste artigo estudamos um caso particular dos problemas de corte, denominado problema bidimensional guilhotinado restrito (PGR). O PGR é um problema NP-difícil que aparece em diversos processos industriais de corte de chapas retangulares, em particular, na indústria de vidro e placas de circuito impresso. Para resolvê-lo, exploramos uma variação do método exato de CHRISTOFIDES \& HADJICONSTANTINOU (1995), baseada numa relaxação do espaço de estados de uma formulação de programação dinâmica do PGR, num procedimento do tipo otimização do subgradiente, $e$ numa heurística de factibilização. $O$ resultado é um método sem garantia de otimalidade, porém bem mais rápido e capaz de resolver problemas maiores do que o método exato de Christofides $e$ Hadjiconstantinou. O desempenho computacional do método é avaliado resolvendo-se diversos exemplos da literatura e exemplos aleatórios, e comparando-se as soluções obtidas com as de CHRISTOFIDES \& HADJICONSTANTINOU (1995) e da conhecida heurística de WANG (1983).

Palavras-chave: problema de corte, padrões de corte bidimensionais guilhotinados restritos, programação dinâmica, heurísticas.

\section{Introdução}

$\mathrm{O}_{a}$ problema de corte consiste em determinar a "melhor" forma de cortar unidades de material (aqui denominadas objetos), de maneira a produzir um conjunto de unidades menores (itens). Esse problema aparece em diversos processos industriais de corte onde os objetos, 
em geral disponíveis em estoque, correspondem a barras de aço, bobinas de papel e alumínio, placas metálicas e de madeira, chapas de vidro e fibra de vidro, peças de couro, etc., e os itens, com dimensões especificadas, são em geral encomendados através de uma carteira de pedidos de clientes. Centenas de artigos foram publicados nas literaturas de pesquisa operacional e gestão da produção tratando problemas de corte, conforme mostram os exames e edições especiais em DYCKHOFF (1992), DOWSLAND \& DOWSLAND (1992), DYCKHOFF \& FINKE (1992), SWEENEY \& PATERNOSTER (1992), MORABITO \& ARENALES (1992), BISCHOFF \& WAESCHER (1995), MUKHACHEVA (1997), DYCKHOFF et al. (1997), e ARENALES et al. (1999).

No presente artigo, tratamos um caso particular dos problemas de corte, denominado problema bidimensional guilhotinado restrito (PGR). Nossa motivação para estudá-lo é que, além de ser um problema de difícil solução exata, ele é importante em diversos processos industriais de corte de chapas retangulares, por exemplo, no corte de chapas de fibra de vidro (objetos) para produção de placas de circuito impresso (itens) encomendadas por clientes (SILVEIRA, 1999). O PGR é bidimensional porque envolve duas dimensões relevantes para a solução (comprimento e largura dos objetos e itens), é guilhotinado porque, devido às restrições impostas pelos equipamentos, os cortes devem ser do tipo guilhotinado (um corte é guilhotinado se, ao ser realizado sobre um retângulo, produzir dois retângulos), e é restrito porque há limitações para o número máximo de vezes que um item poderá ser cortado a partir de um objeto, além das limitações impostas pelas dimensões físicas do objeto.

Ao tratar o PGR, a maioria dos autores propôs métodos aproximados. Exemplos disso podem ser encontrados em WANG (1983), que propôs uma heurística que combina itens em arranjos horizontais e verticais para formar padrões de corte; em VASKO (1989) e OLIVEIRA \& FERREIRA (1990), que apresentaram refinamentos do algoritmo de Wang; em MORABITO
\& ARENALES (1996), que propuseram uma abordagem baseada numa busca em um grafo-e/ou, em MORNAR \& KHOSHNEVIS (1997), que adaptaram o algoritmo de Wang para uma aplicação particular de cortes de placas de circuito impresso, e em FAYARD et al. (1998), que apresentaram um algoritmo baseado em problemas da mochila unidimensionais para combinar faixas horizontais e verticais dentro de sub-retângulos.

Poucos autores apresentaram métodos exatos para o PGR, entre eles, VISWANATHAN \& BAGCHI (1993), que propuseram um procedimento de busca em árvore baseado na estratégia de busca o melhor primeiro; HIFI (1997), que sugeriu um melhoramento no algoritmo de Viswanathan e Bagchi; CHRISTOFIDES \& WHITLOCK (1977), que apresentaram um algoritmo de busca em árvore que utiliza uma rotina baseada no problema de transporte para obter limitantes durante a busca; e CHRISTOFIDES \& HADJICONSTANTINOU (1995), que também propuseram um algoritmo de busca em árvore, porém utilizando limitantes derivados de uma relaxação do espaço de estados de uma formulação de programação dinâmica, e um procedimento do tipo otimização do subgradiente (ascensão no espaço de estados). Tal método é mais eficaz do que o de CHRISTOFIDES \& WHITLOCK (1977). CHRISTOFIDES \& HADJICONSTANTINOU (1995) não chegaram a aplicar este método para resolver exemplos reais de processos de corte, mas apenas para resolver alguns exemplos da literatura e exemplos gerados aleatoriamente, todos de tamanho moderado.

Neste artigo exploramos uma variação do método de CHRISTOFIDES \& HADJICONSTANTINOU (1995), onde, ao invés de aplicarmos o algoritmo de busca em árvore, optamos por desenvolver uma simples heurística de factibilização que, em cada iteração do procedimento de otimização do subgradiente, utiliza a solução relaxada de programação dinâmica para tentar acentuar a melhor solução factível obtida até então. O resultado é um método sem garantia de otimalidade, porém, 
bem mais rápido e capaz de resolver problemas maiores do que o método exato de CHRISTOFIDES \& HADJICONSTANTINOU (1995). O desempenho computacional do método é ilustrado resolvendo-se alguns exemplos da literatura e exemplos aleatórios, e comparando-se as soluções obtidas com as soluções ótimas do método de CHRISTOFIDES \& HADJICONSTANTINOU (1995) e com as soluções produzidas por métodos heurísticos, entre eles, o algoritmo de Wang (esta é a heurística mais referenciada na literatura para resolver o PGR).

Os resultados destes experimentos mostram que uma das vantagens do presente método sobre outros métodos heurísticos é que, nos casos em que ele encontra uma solução ótima, em geral também fornece um certificado de otimalidade. Além disso, nos casos em que a solução obtida é subótima, o método é capaz de produzir um excelente limitante superior para o valor da solução ótima do PGR, o que permite uma boa estimativa do gap de otimalidade. Convém salientar que, na maioria dos exemplos aqui analisados, este limitante igualou-se ou ficou muito perto do valor da solução ótima.

Este trabalho está organizado da seguinte maneira: na próxima seção discutimos a modelagem do PGR e o método de solução aqui proposto baseado em CHRISTOFIDES \& HADJICONSTANTINOU (1995), e na seção 3 analisamos os resultados computacionais do método, comparando-os com os dos outros métodos. Finalmente, na seção 5, apresentamos as conclusões e perspectivas para pesquisa futura.

\section{Modelagem do PGR e Método de Solução}

$\mathrm{P}$ roblemas de corte têm sido abordados na literatura principalmente por: (i) programação linear combinada com uma técnica de geração de colunas (padrões de corte) (GILMORE \& GOMORY, 1965), e (ii) heurística gulosa de aspiração da demanda, combinada com um procedimento de geração de padrões de corte (HINXMAN, 1980). A abordagem (i) pode ser apropriada quando o problema de corte é irrestrito, isto é, não há limitações para o número máximo de vezes em que um tipo de peça poderá ser cortado a partir de uma placa, além das limitações impostas pelas dimensões físicas da placa. Uma heurística gulosa de aspiração da demanda bastante simples é a chamada redução exaustiva repetitiva (HINXMAN, 1980):

\section{Heurística de aspiração:}

Enquanto a demanda de peças não for cumprida, faça-se:

Passo 1: Gere o melhor padrão de corte para o PGR.

Passo 2: Repita este padrão, tanto quanto for possível, em função da demanda das peças. Ou seja, corte no maior número de vezes possível este padrão, levando em conta a demanda atual das peças deste padrão.

Passo 3: Atualize a demanda das peças, ou seja, retire da demanda atual a quantidade de peças que foi cortada no passo 2. Eventualmente, inclua na demanda as peças de novos pedidos.

Podemos verificar que a maior dificuldade da heurística de aspiração se encontra no passo 1, que é o foco deste trabalho. A seguir, descrevemos uma formulação de programação dinâmica para resolver o PGR do passo 1 .

\subsection{Formulação de programação dinâmica}

Seja $R_{0}$ o retângulo (chapa) de comprimento e largura $L \times W$, e $R$ um conjunto de $n$ retângulos menores (peças) $R_{1}, R_{2}, \ldots, R_{n}$, com comprimentos e larguras $l_{1}, \times w_{1}, l_{2}, \times w_{2}, \ldots, l_{n} \times w_{n}$, demandas $b_{1}, b_{2}, \ldots, b_{n}$, e valores $v_{1}, v_{2}, \ldots, v_{n}$. O PGR consiste em encontrar o padrão de corte guilhotinado para $R_{0}$ com o maior valor total possível, usando-se não mais do que $b_{\mathrm{i}}$ réplicas de cada retângulo $R_{i}, i=1,2, \ldots, n$ (uma vez que temos a restrição de não gerar estoque, ou seja, podemos produzir no máximo $b_{i}$ réplicas de cada retângulo $R_{i}$ ). Se todos os valores $v_{i}$ forem iguais 


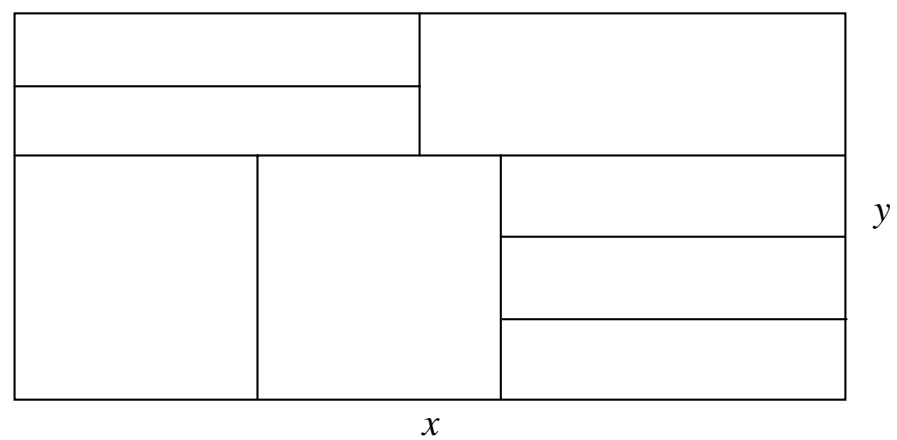

Figura 1 - Exemplo de padrão de corte para $(x, y)$ em 3-estágios

às áreas $l_{i} w_{i}$, então o objetivo corresponde a minimizar a perda de material. Admitimos que os comprimentos e larguras da chapa e das peças são números inteiros e, por simplicidade, que peças $l_{i} \times w_{i}$ são diferentes de peças $w_{i} \times l_{i}$ (casos em que as peças podem ser rotacionadas podem ser tratados por meio de simples modificações nas definições de $F_{0}$ em (3) e (7), e $X$ e $Y$ em (11) e (12) adiante).

Para desenvolver uma fórmula recursiva de programação dinâmica, admitimos que os cortes são produzidos em estágios. A Figura 1 ilustra um padrão de corte em 3 estágios para um retângulo $(x, y)$, onde, no primeiro estágio, um corte horizontal divide o retângulo $(x, y)$ em 2 retângulos (superior e inferior); no segundo estágio, um corte vertical divide o retângulo superior em dois retângulos (superior esquerdo e superior direito), e dois cortes verticais dividem o retângulo inferior em três retângulos (inferior esquerdo, inferior intermediário e inferior direito); e, no terceiro estágio, um corte horizontal divide o retângulo superior esquerdo em dois retângulos, e dois cortes horizontais dividem o retângulo inferior direito em três retângulos. Note que os cortes de cada estágio são sempre paralelos aos eixos $x$ ou $y$, e que os cortes de dois estágios consecutivos são sempre perpendiculares entre si.

Sejam $s_{1}, s_{2}, \ldots, s_{n}$ as quantidades de peças dos tipos $1,2, \ldots, n$ que podem ser usadas para produzir um padrão de corte factível para o retângulo $(x, y)$. Representamos essas peças pela seqüência ordenada:

$$
S_{x y}=\left\{s_{1}, s_{2}, \ldots, s_{n}\right\}
$$

Note que, para a chapa $(L, W)$, podemos usar quaisquer peças do conjunto $R$, e assim:

$$
S_{L W}=\left\{b_{1}, b_{2}, \ldots, b_{n}\right\}
$$

Definimos $F_{k}\left(x, y, S_{x y}\right)$ como o valor do padrão de corte ótimo em até $k$-estágios, para um retângulo de tamanho $(x, y)$, usando uma combinação factível de retângulos do conjunto $S_{x y}$, com cortes no primeiro estágio paralelos ao eixo $y$. Similarmente, definimos $G_{k}\left(x, y, S_{x y}\right)$ como o valor do padrão de corte ótimo em até $k$-estágios, para um retângulo de tamanho $(x, y)$, usando uma combinação factível de retângulos do conjunto $S_{x y}$, com cortes no primeiro estágio paralelos ao eixo $x$. Sejam $X=\{1,2, \ldots, L\}$ e $Y=\{1,2, \ldots, W\}$. As funções recursivas $F_{k}\left(x, y, S_{x y}\right)$ e $G_{k}\left(x, y, S_{x y}\right)$, para todo $k \geq 1, x \in X, y \in Y$ e $S_{x y} \subseteq S_{L W}$, são definidas como (CHRISTOFIDES \& HADJICONSTANTINOU, 1995):

$$
\begin{aligned}
& F_{k}\left(x, y, S_{x y}\right)=\max \left[\begin{array}{c}
G_{k-1}\left(x, y, S_{x y}\right), \\
\max _{\substack{x^{\prime}<x \\
x^{\prime} \in X, S^{\prime} \subset S_{x y}}}\left[\begin{array}{c}
F_{k}\left(x^{\prime}, y, S^{\prime}\right)+ \\
+G_{k-1}\left(x-x^{\prime}, y, S_{x y}-S^{\prime}\right)
\end{array}\right]
\end{array}\right] \\
& G_{k}\left(x, y, S_{x y}\right)=\max \left[\begin{array}{c}
F_{k-1}\left(x, y, S_{x y}\right), \\
\max _{\substack{y^{\prime}<y \\
y^{\prime} \in Y, S^{\prime} \subset S_{x y}}}\left[\begin{array}{c}
G_{k}\left(x, y^{\prime}, S^{\prime}\right)+ \\
+F_{k-1}\left(x, y-y^{\prime}, S_{x y}-S^{\prime}\right)
\end{array}\right]
\end{array}\right]
\end{aligned}
$$




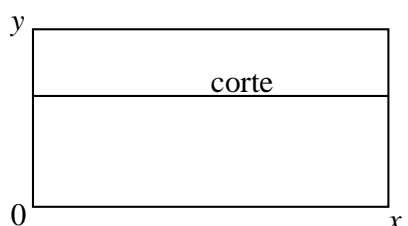

(a)

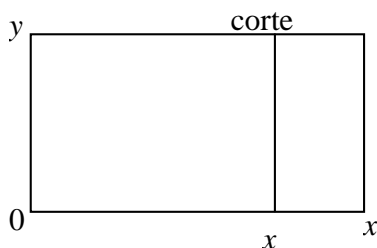

(b)

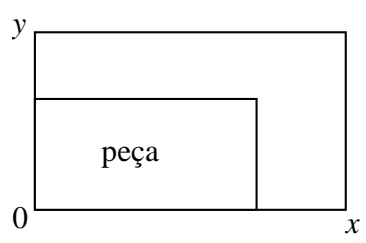

(c)

Figura 2 - (a) Nenhum corte neste estágio, mas um corte no estágio anterior paralelo ao eixo $x$, (b) corte neste estágio paralelo ao eixo $y$, (c) alocação de uma peça no retângulo $(x, y) \mathrm{em} k=0$

O primeiro termo do colchete da fórmula (1) corresponde ao caso em que, no primeiro estágio do padrão ótimo em até $k$ estágios de $(x, y)$, não há cortes em $(x, y)$ paralelos ao eixo $y$, o que implica que este padrão pode ser considerado como um padrão em até $k-1$ estágios, com os cortes do primeiro estágio paralelos ao eixo $x$, como na Figura 2(a). O segundo termo do colchete da fórmula (1) corresponde ao caso em que, no primeiro estágio do padrão ótimo em até $k$ estágios de $(x, y)$, existe pelo menos um corte em $(x, y)$ paralelo ao eixo $y$, digamos um corte vertical $x^{\prime} \in X, x^{\prime}<x$, produzindo dois retângulos $(x, y)$ e $(x-x, y)$, como na Figura 2(b). Neste caso, temos dois padrões de corte associados com estes retângulos. No primeiro, as peças pertencem ao conjunto $S^{\prime}$, os cortes no primeiro estágio do padrão ótimo de $(x, y)$ são paralelos ao eixo $y$, e ainda há $k$ estágios no total (dado que pode haver outros cortes verticais $x$ " $<x$ ' em $(x, y)$, que não implicam em outro estágio em $(x, y))$. No segundo, as peças pertencem ao conjunto complementar $S_{x y}-S$, os cortes no primeiro estágio do padrão ótimo de $(x-x, y)$ são paralelos ao eixo $x$, e há somente $k-1$ estágios no total (dado que cortes horizontais em $(x-x, y)$ implicam em outro estágio em $(x, y))$. Similarmente para a fórmula (2).

Um valor para $k=0$ em $F_{k}\left(x, y, S_{x y}\right)$ ou $G_{k}\left(x, y, S_{x y}\right)$ (fórmulas (1) e (2)) corresponde a uma alocação da maior peça (i.e., mais valiosa) do conjunto $S_{x y}$ para o retângulo $(x, y)$ (ver Figura 2(c)). Os dois cortes para produzir esta peça a partir de $(x, y)$ não são considerados como estágios adicionais de corte. Assim, condições iniciais são fornecidas, para todo $x, y$ e $S_{x y}$, por:

$$
\begin{gathered}
F_{0}\left(x, y, S_{x y}\right)=\max \left[0, \max _{i \in S_{x y}}\left[v_{i} \mid l_{i} \leq x, w_{i} \leq y\right]\right], \\
G_{0}\left(x, y, S_{x y}\right)=F_{0}\left(x, y, S_{x y}\right) .
\end{gathered}
$$

Note que o máximo entre $F_{k}\left(x, y, S_{x y}\right)$ e $G_{k}\left(x, y, S_{x y}\right)$ em (1)-(4) corresponde à solução ótima do PGR $k$-estágios para o retângulo $(x, y)$, gerado pelo uso das peças em $S_{x y}$, quando a direção dos cortes do primeiro estágio não é especificada. Em particular, $\max \left[F_{k}\left(L, W, S_{L W}\right)\right.$, $\left.G_{k}\left(L, W, S_{L W}\right)\right]$ corresponde à solução ótima do PGR $k$-estágios para a chapa $R_{0}=L \times W$. Se não for especificado um número máximo de estágios (i.e., PGR multiestágios), devemos considerar o valor de $k$ como sendo aquele em que:

$$
\begin{gathered}
F_{k}\left(L, W, S_{L W}\right)=F_{k+1}\left(L, W, S_{L W}\right) \\
\mathrm{e} \\
G_{k}\left(L, W, S_{L W}\right)=G_{k+1}\left(L, W, S_{L W}\right)
\end{gathered}
$$

A principal dificuldade desta formulação é a dimensão do espaço de estados associada principalmente à variável de estado $S_{L W}$.

A seguir, discutimos uma relaxação do espaço de estados da formulação (1)-(4), combinada com um procedimento do tipo otimização do subgradiente (ascensão no espaço de estados), conforme explorado em CHRISTOFIDES \& HADJICONSTANTINOU (1995), que produz bons limitantes para o problema original. Em seguida, desenvolvemos uma heurística de factibilização que, em cada iteração do procedimento de otimização do subgradiente, utiliza a solução relaxada para produzir boas soluções factíveis para o problema original. Convém salientar que 


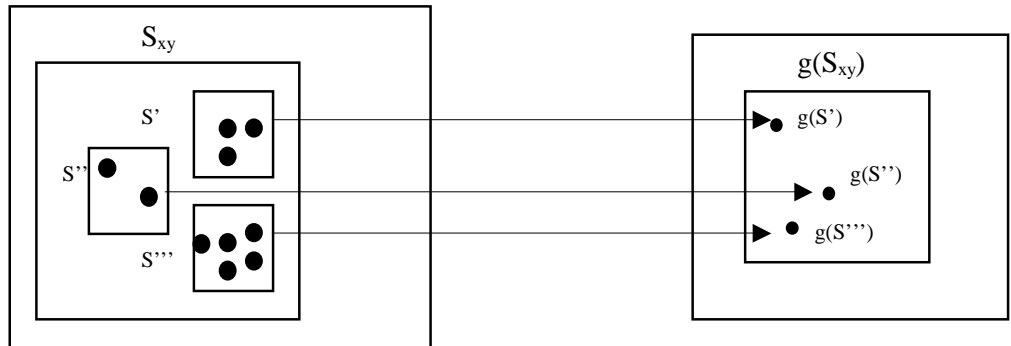

Figura 3 - Representação da relaxação do espaço de estados

\section{CHRISTOFIDES \& HADJICONSTANTINOU}

(1995) não exploraram heurísticas de factibilização; ao contrário, aplicaram os limitantes obtidos conforme discussão acima num procedimento de busca em árvore, para garantir encontrar uma solução ótima para o problema.

\subsection{Relaxação do espaço de estados}

Considere-se a formulação (1)-(4). Seja $g($. uma função de mapeamento escalar inteira, do domínio de estados $\left(x, y, S_{x y}\right)$ para algum outro domínio $\left(x, y, g\left(S_{x y}\right)\right)$, com menor cardinalidade. Por exemplo, seja $g\left(S_{x y}\right)=\sum_{s_{i} \in S_{x y}} s_{i} ; \log 0$, todos os subconjuntos $S^{\prime} \subset S_{x y}$ com mesmo valor $g\left(S^{\prime}\right)$ (i.e., com mesma quantidade total de peças disponíveis) são representados por apenas um ponto no novo domínio (Figura 3). Portanto, trata-se de uma relaxação do espaço de estados original.

A fórmula recursiva (1) para o problema de corte pode agora ser reescrita para o novo espaço relaxado, para todo $k \geq 1, x \in X, y \in Y$ e $g\left(S_{x y}\right)$, como segue:

$$
\begin{aligned}
& F_{k}\left(x, y, g\left(S_{x y}\right)\right)= \\
& =\max \left[\begin{array}{c}
G_{k-1}\left(x, y, g\left(S_{x y}\right)\right), \\
\max _{\substack{x^{\prime}<x, x^{\prime} \in X \\
g\left(S^{\prime}<g\left(S_{x y}\right)\right.}}\left[\begin{array}{c}
F_{k}\left(x^{\prime}, y, g\left(S^{\prime}\right)\right)+ \\
+G_{k-1}\left(x-x^{\prime}, y, g\left(S_{x y}-S^{\prime}\right)\right)
\end{array}\right]
\end{array}\right]
\end{aligned}
$$

Similarmente para a fórmula recursiva (2). Como se trata de uma relaxação da formulação (1)-(2), temos que:

$$
\begin{gathered}
F_{k}\left(x, y, g\left(S_{x y}\right)\right) \geq F_{k}\left(x, y, S_{x y}\right) \\
\mathrm{e} \\
G_{k}\left(x, y, g\left(S_{x y}\right)\right) \geq G_{k}\left(x, y, S_{x y}\right)
\end{gathered}
$$

e assim, $\max \left[F_{k}\left(x, y, g\left(S_{x y}\right)\right), G_{k}\left(x, y, g\left(S_{x y}\right)\right)\right]$ produz limitantes superiores para a solução ótima do PGR com até $k$-estágios (uma prova formal disto pode ser encontrada em CHRISTOFIDES et al., 1981). Obviamente, é desejável que a função $g$ seja fácil de ser computada, e que tenha sua contrapartida muito menor do que seu domínio. CHRISTOFIDES \& HADJICONSTANTINOU (1995) propuseram associar um peso inteiro não negativo $q_{i}$ a cada peça $i$ no conjunto $R$, e definiram $g$ como:

$$
g\left(S_{x y}\right)=q \equiv \sum_{s_{i} \in S_{x y}} s_{i} q_{i} \quad \text { e } \quad g\left(S^{\prime}\right)=q^{\prime} \equiv \sum_{s_{i} \in S^{\prime}} s_{i} q_{i}
$$

Logo, $g\left(S_{x y}-S^{\prime}\right)$ pode ser facilmente computada por $q-q^{\prime}$, e as fórmulas (1)-(4) são reescritas por:

$$
F_{k}(x, y, q)=\max \left[\begin{array}{c}
G_{k-1}(x, y, q), \\
\max _{\substack{x^{\prime}<x, x, x^{\prime} \in X, q^{\prime}=0, \ldots, q}}\left[\begin{array}{c}
F_{k}\left(x^{\prime}, y, q^{\prime}\right)+ \\
+G_{k-1}\left(x-x^{\prime}, y, q-q^{\prime}\right)
\end{array}\right]
\end{array}\right]
$$

$$
G_{k}(x, y, q)=\max \left[\begin{array}{c}
F_{k-1}(x, y, q), \\
\max _{\substack{y^{\prime}<y, y \in Y, q^{\prime}=0, \ldots, q}}\left[\begin{array}{c}
G_{k}\left(x, y^{\prime}, q^{\prime}\right)+ \\
+F_{k-1}\left(x, y-y^{\prime}, q-q^{\prime}\right)
\end{array}\right]
\end{array}\right]
$$




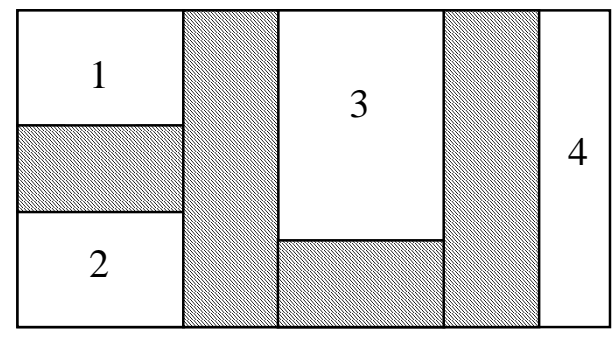

(a)

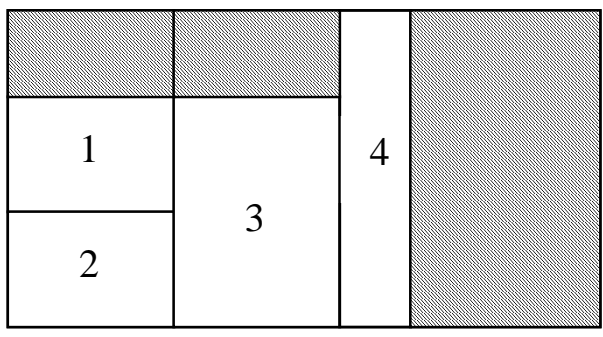

(b)

Figura 4 - (a) Padrão de corte não normalizado, (b) padrão de corte normalizado

e inicializadas por

$F_{0}(x, y, q)=\max \left[0, \max _{i=1, \ldots, n}\left[v_{i} \mid l_{i} \leq x, w_{i} \leq y, q_{i} \leq q\right]\right]$,

$$
G_{0}(x, y, q)=F_{0}(x, y, q) .
$$

As recursões (5)-(8), aplicadas para todo $k \geq 1, x \in X, y \in Y$ e $q=0, \ldots, Q$, onde

$$
Q=\sum_{b_{i} \in S_{L W}} b_{i} q_{i},
$$

podem ser usadas para obtermos um limitante superior $Z_{U B}$ para o valor da solução ótima do PGR $k$-estágios, para um dado conjunto de pesos $q_{1}, q_{2}, \ldots, q_{n}$. Este limitante é calculado por:

$$
Z_{U B}=\max \left[F_{k}(L, W, Q), G_{k}(L, W, Q)\right]
$$

Note que para $q_{1}=0, q_{2}=0, \ldots, q_{n}=0$ e $k$ suficientemente grande, o limitante $Z_{U B}$ em (10) corresponde à solução ótima do problema guilhotinado irrestrito. A computação de $Z_{U B}$ pode ser reduzida pela restrição aos padrões de cortes normais (HERZ, 1972; CHRISTOFIDES \& WHITLOCK, 1977). Qualquer padrão de corte é dito normalizado se qualquer peça cortada tem sua margem do lado esquerdo e sua margem inferior adjacentes a outra peça cortada ou às bordas de $R_{0}$, como mostra a Figura 4. Uma conseqüência disso é que os conjuntos $X=\{1,2, \ldots, L\}$ e $Y=\{1,2, \ldots, W\}$ em (5)-(6) podem ser significativamente reduzidos, sem perda de generalidade, ao serem redefinidos por:

$$
X=\left\{\begin{aligned}
& x \mid x=\sum_{i=1}^{n} \theta_{i} l_{i}, 1 \leq x \leq L, 0 \leq \theta_{i} \leq b_{i} \\
& e \theta_{i} \text { inteiro, } i=1, \ldots, n
\end{aligned}\right\}
$$

$$
Y=\left\{\begin{array}{r}
y \mid y=\sum_{i=1}^{n} \tau_{i} w_{i}, 1 \leq y \leq W, 0 \leq \tau_{i} \leq b_{i} \\
e \tau_{i} \text { inteiro, } i=1, \ldots, n
\end{array}\right\}
$$

Neste caso, as fórmulas recursivas (5) e (6), aplicadas para todo $k \geq 1, x \in X, y \in Y$ e $q=0, \ldots, Q$ (conforme (9), (11) e (12)), são reescritas por:

$F_{k}(x, y, q)=\max \left[\begin{array}{c}G_{k-1}(x, y, q), \\ \max _{\substack{x^{\prime}<x, x^{\prime} \in X, \ldots \\ q^{\prime}=0, \ldots, q}}\left[\begin{array}{c}F_{k}\left(x^{\prime}, y, q^{\prime}\right)+ \\ \left.+G_{k-1}\left(x-x^{\prime}\right], y, q-q^{\prime}\right)\end{array}\right]\end{array}\right]$

$G_{k}(x, y, q)=\max \left[\begin{array}{c}F_{k-1}(x, y, q), \\ \max _{\substack{y^{\prime}<y, y^{\prime} \in Y, q^{\prime}=0, \ldots, q}}\left[\begin{array}{c}G_{k}\left(x, y^{\prime}, q^{\prime}\right)+ \\ +F_{k-1}\left(x,\left\lfloor y-y^{\prime}\right\rfloor, q-q^{\prime}\right)\end{array}\right]\end{array}\right]$

onde $\lfloor x\rfloor=\max \left\{x_{1} \mid x_{1} \leq x, x_{1} \in X \cup\{0\}\right\}$, $\lfloor y\rfloor=\max \left\{y_{1} \mid y_{1} \leq y, y_{1} \in Y \cup\{0\}\right\} \quad$ (estas definições são necessárias para garantirmos que o resultado das diferenças $x-x^{\prime}$ e $y-y^{\prime}$ em (13) e (14) pertençam aos conjuntos $X$ e $Y$ em (11) e (12)), e $F_{k}(x, y, q)=G_{k}(x, y, q)=0$ se $x=0$ ou $y=0$ (note agora que, por causa das 
definições de $\lfloor x\rfloor$ e $\lfloor y\rfloor$, podemos ter $x=0$ ou $y=0$ ). As fórmulas (13)-(14) são inicializadas conforme antes, por (7)-(8).

Uma vez computado o valor do limitante $Z_{U B}$ em (10) para um dado conjunto de pesos $q_{1}, q_{2}, \ldots, q_{n}$, e $k$ suficientemente grande, é necessário fazer um backtracking para determinar as peças em $R$ que compõem o padrão correspondente (não necessariamente factível) para a chapa $R_{0}$. Isso requer o uso de ponteiros que indicam, para cada conjunto de valores $(k, x, y, q)$, os termos na recursão (13)-(14) que levaram aos valores de $F_{k}(x, y, q)$ e $G_{k}(x, y, q)$. Uma simples rotina backtracking pode ser definida para determinar a sequiência de cortes realizados sobre a chapa $R_{0}$, em conjunto com uma estrutura de dados apropriada para representar a lista de peças em $R$ produzidas durante o processo de corte. Seja $\gamma_{i}$ o número de vezes que a peça $i$ foi usada neste padrão. Se $\gamma_{i} \leq b_{i}$ para todo $i=1, \ldots, n$, então uma solução factível para o PGR multiestágios foi encontrada, e logo é ótima. Caso contrário, um procedimento do tipo otimização do subgradiente pode ser utilizado para otimizar o limitante $Z_{U B}$ em (10), variando-se os pesos $q_{1}, q_{2}, \ldots, q_{n}$.

\subsection{Ascensão no espaço de estados}

Um método de modificação do espaço de estados, associado com a formulação relaxada (13)-(14), pode ser usado numa tentativa de minimizar o limitante $Z_{U B}$ em (10). O objetivo desta técnica é penalizar as infactibilidades de modo a forçar que a solução do problema relaxado se aproxime cada vez mais da factibilidade. Sejam $q$ o vetor de pesos $\left(q_{1}, q_{2}, \ldots, q_{n}\right)$, $f(q)$ o limitante superior $Z_{U B}$ produzido por (10) usando o vetor $q$, e $D$ a máxima dimensão do espaço de estados relaxado que desejamos modificar (isto é, os maiores valores de $Q$ em (9)). Temos que resolver o seguinte problema:

$$
\begin{aligned}
& f\left(q^{*}\right)=\min _{q \geq 0}[f(q)] \\
& \text { s.a.: } \quad \sum_{b_{i} \in S_{L W}} b_{i} q_{i} \leq D
\end{aligned}
$$

A minimização de $f(q)$ no problema acima é, em geral, difícil, dado que $f(q)$ é uma função descontínua em $q$. Entretanto, um procedimento do tipo "otimização do subgradiente" para modificar os pesos $q_{i}$ e ascender no espaço de estados pode ser usado (CHRISTOFIDES \& HADJICONSTANTINOU, 1995). O procedimento é baseado no seguinte princípio: considere-se $\gamma_{i}$ conforme definido anteriormente. Se $\gamma_{i} \leq b_{i}$ para todo $i=1, \ldots, n$, então o problema está resolvido; caso contrário, então é razoável tentar reduzir a diferença $\gamma_{i}-b_{i}$ para se mover em direção à factibilidade. Um simples modo de se conseguir isso é incrementar o peso $q_{i}$ das peças para as quais $\gamma_{i}>b_{i}$ e, ao mesmo tempo, decrementar o peso $q_{i}$ das peças para as quais $\gamma_{i} \leq b_{i}$.

Neste trabalho utilizamos a seguinte fórmula para atualizar os valores dos pesos $q_{i}$ :

$q_{i}=\left\{\begin{array}{cc}q_{i}+\left\lfloor t \sqrt{\left(\gamma_{i}-b_{i}\right)} \quad\right. & \text { se } \gamma_{i}>b_{i,} \\ \max \left[0, q_{i}-\left\lfloor t \sqrt{\left(b_{i}-\gamma_{i}\right)}\right\rfloor\right] & \text { se } \gamma_{i} \leq b_{i,}\end{array}\right.$

onde $\lfloor z\rfloor$ denota o maior inteiro menor ou igual a $z$, e $t$ é o tamanho do passo escalar positivo, dado por:

$$
t=\max \left[1, \sqrt{\pi\left(Z_{U B}-Z_{L B}\right) / \sum_{i=1}^{m}\left(b_{i}-\gamma_{i}\right)^{2}}\right]
$$

Note que esta fórmula, similar ao tamanho dos passos na otimização do subgradiente (HELD et al., 1974), é uma pequena modificação da utilizada em CHRISTOFIDES \& HADJICONSTANTINOU (1995). Nossa motivação para o limite inferior $t=1$ é permitir que $q_{i}$ em (15) seja alterado sempre que $\gamma_{i} \neq b_{i}$. O parâmetro $\pi$ é inicialmente fixado em 2,0 , sendo então reduzido pela metade a cada 3 iterações, até que $\pi$ seja inferior a $\varepsilon=0,05$ (i.e., após 18 iterações). $Z_{L B}$ é o valor de um limitante inferior (correspondendo a uma solução factível) para a solução ótima do PGR, inicializado por alguma heurística. $Z_{U B}$ é o valor do limitante superior 
corrente em (10). Note que, se $Z_{U B}=Z_{L B}$, então o procedimento de otimização do subgradiente pode ser interrompido, com $Z_{L B}$ sendo a solução ótima para o problema original.

\section{Algoritmo de otimização do subgradiente:}

Passo 1: Determine $X$ e $Y$ em (11)-(12), e escolha valores iniciais para $Z_{L B}$ e para o vetor $q$, por exemplo, $q_{i}=0$, $i=1, \ldots, n$.

Passo 2: Resolva a formulação relaxada (13)(14), com $k$ suficientemente grande, para obter o valor ótimo $Z_{U B}$ em (10) relativo ao vetor $q$ atual.

Passo 3: Cheque se o padrão de corte associado $\operatorname{com} Z_{U B}$ gera uma solução factível para o PGR. Se sim, então esta solução é ótima e o procedimento é terminado. Caso contrário, se $Z_{U B}<Z_{\min }$ (o mínimo limitante superior obtido até então), atualize $Z_{\text {min }} \operatorname{com} Z_{\min }=Z_{U B}$.

Passo 4: Execute uma heurística de factibilização (descrita a seguir) para encontrar uma solução factível para o problema. Se o valor desta solução for maior que $Z_{L B}$ (o valor da melhor solução factível obtida até então), atualize $Z_{L B}$. Se $Z_{L B}=Z_{\text {min }}$, então esta solução é ótima e o procedimento é terminado.

Passo 5: Modifique o vetor $q$ usando a fórmula dada em (15), e atualize $Q$ em (9) e $\pi$.

Passo 6: Volte para o passo 2 com o novo vetor $q$, a menos que um número suficiente de iterações já tenha sido realizado, ou $\pi \leq \varepsilon$.

No final do algoritmo, a solução ótima para o PGR pode ter sido encontrada (passo 3 ou 4). Caso contrário, a solução apresentada é a melhor obtida no passo 4 com a heurística de factibilização, que não tem garantia de otimalidade. Em CHRISTOFIDES \& HADJICONSTANTINOU (1995), não há o passo 4, e o melhor limitante superior $Z_{\text {min }}$ obtido pelo algoritmo é usado num procedimento de busca em árvore para resolver otimamente o problema.

\subsection{Heurística de factibilização}

Uma heurística de factibilização bastante simples é resumida nos seguintes passos:

Passo 1: Retire aleatoriamente do padrão as peças que excederam a demanda atual. Por exemplo, se o padrão contém três peças do tipo 1, e apenas duas peças do tipo 1 são demandadas, então retire aleatoriamente do padrão uma peça do tipo 1.

Passo 2: Para cada "buraco" produzido no passo 1 (i.e., espaço formado pelas peças que foram retiradas do padrão), determine a solução homogênea (arranjo com peças do mesmo tipo) mais valiosa, dentre os tipos de peças que ainda não excederam a demanda. Guarde o valor da melhor solução homogênea obtida para cada buraco.

Passo 3: Preencha o buraco que resultar no maior valor para o padrão. Atualize a demanda das peças utilizadas para preencher este buraco.

Passo 4: Volte para o passo 2 enquanto existirem buracos que ainda possam ser preenchidos por peças demandadas.

Esta heurística é executada no passo 4 do algoritmo de otimização do subgradiente (seção 2.3). Ao término do algoritmo, se tivermos encontrado uma solução factível com valor $Z_{\text {min }}$, então esta solução é ótima. Caso contrário, o algoritmo retorna a melhor solução produzida pela heurística de factibilização ao longo de todas as iterações, junto com o limitante inferior $Z_{\text {min }}$ para o valor da solução ótima do PGR.

\section{Resultados Computacionais}

$\mathrm{N}$ esta seção apresentamos alguns resultados computacionais para ilustrar o desempenho computacional do método proposto na seção 2 . Tanto as implementações deste método quanto as do algoritmo proposto em WANG (1983) foram codificadas em linguagem Pascal (compilador 
Tabela 1 - Resultados computacionais para 6 exemplos da literatura

\begin{tabular}{|c|c|c|c|c|c|c|c|c|c|c|c|c|c|}
\hline \multirow{2}{*}{ Prob } & \multicolumn{4}{|c|}{ Dados } & \multicolumn{2}{|c|}{ Wang } & \multicolumn{3}{|c|}{ Christ } & \multicolumn{2}{|c|}{ Christ/Fact } & \multirow{2}{*}{$\frac{\text { Ótima }}{\text { Sol. }}$} & \multirow{2}{*}{$\frac{\text { Melh }}{\%}$} \\
\hline & $(L, W)$ & $n$ & $|X|$ & $|Y|$ & Sol. & Tетро & Lim. & Sol. & Tempo & Sol. & Teтро & & \\
\hline$C W 1$ & $(15,10)$ & 7 & 12 & 10 & $244^{*}$ & $<1$ & 244 & $244 *$ & $<1$ & $244^{*}$ & $<1$ & 244 & 0,0 \\
\hline$C W 2$ & $(40,70)$ & 10 & 29 & 56 & 2732 & $<1$ & 2892 & 2732 & 214 & 2732 & 219 & 2892 & 0,0 \\
\hline$C W 3$ & $(40,70)$ & 20 & 25 & 55 & 1400 & $<1$ & 1860 & 1400 & 199 & $1860^{*}$ & 218 & 1860 & 32,9 \\
\hline$O F 1$ & $(70,40)$ & 10 & 48 & 32 & 2172 & $<1$ & 2737 & $2737 *$ & 341 & $2737 *$ & 164 & 2737 & 26,0 \\
\hline$O F 2$ & $(70,40)$ & 10 & 41 & 32 & 2083 & $<1$ & 2717 & 2083 & 249 & 2506 & 206 & 2690 & 20,3 \\
\hline$W A$ & $(70,40)$ & 20 & 55 & 25 & 2277 & $<1$ & 2721 & $2721^{*}$ & 5 & $2721^{*}$ & 3 & 2721 & 19,5 \\
\hline \multicolumn{5}{|c|}{ Média } & & $<1$ & & & 168 & & 135 & & 16,5 \\
\hline
\end{tabular}

* solução ótima

Delphi 4 da Borland), e foram rodadas num microcomputador Pentium (350 Mhz, 256 Mbytes de memória RAM).

Dividimos os experimentos desta seção em duas partes: exemplos retirados da literatura (seção 3.1) e exemplos gerados aleatoriamente (seção 3.2). Em todos os exemplos resolvidos pelo algoritmo proposto em WANG (1983), usamos como parâmetro $\beta=0,03$, ou seja, permitimos uma perda de no máximo $3 \%$ dentro dos arranjos horizontais e verticais gerados pelo algoritmo. Convém salientar que este foi o valor que apresentou os melhores resultados em WANG (1983). Como o algoritmo de Wang é suficientemente rápido (conforme é visto a seguir, ele consumiu menos de 1 segundo para resolver cada exemplo) e produz soluções relativamente boas, estas foram utilizadas para inicializar o valor de $Z_{L B}$ no passo 1 do algoritmo de otimização do subgradiente (seção 2.3). Desta maneira, os resultados a seguir indicam quanto o segundo algoritmo é capaz de melhorar as soluções do primeiro.

\subsection{Exemplos da literatura}

Tomamos 6 exemplos amplamente utilizados na literatura para comparações de métodos de solução para o PGR: os exemplos $C W 1, C W 2$ e
CW3 em CHRISTOFIDES \& WHITLOCK (1977), OF1 e OF2 em OLIVEIRA \& FERREIRA (1990), e WA em WANG (1983). As soluções ótimas destes exemplos são conhecidas na literatura. A Tabela 1 resume os dados dos exemplos e os resultados computacionais obtidos; os demais dados dos exemplos podem ser encontrados em SILVEIRA (1999). As colunas Wang, Christ e Christ/Fact apresentam, respectivamente, as soluções obtidas pela heurística de WANG (1983), pelo algoritmo de otimização do subgradiente de CHRISTOFIDES \& HADJICONSTANTINOU (1995) (algoritmo da seção 2.3 sem o passo 4), e pelo algoritmo de otimização do subgradiente junto com a heurística de factibilização (algoritmo da seção 2.3 com o passo 4, e algoritmo da seção 2.4). Os tempos computacionais (coluna Tempo) referemse a segundos, e o símbolo "< 1" indica um tempo menor que 1 segundo. A coluna Lim apresenta o melhor limitante superior gerado por Chris e Christ/Fact, e as colunas Ótima e Melh apresentam, respectivamente, a solução ótima e a melhoria (em \%) da solução de Christ/Fact em relação à solução da heurística de Wang.

Observe na Tabela 1 que: (i) enquanto Wang foi capaz de encontrar a solução ótima apenas de $C W 1$, Christ encontrou a solução ótima de $C W 1$, OF1 e WA, e Christ/Fact a solução ótima de 
CW1, CW3, OF1 e WA; (ii) Christ e Christ/Fact produziram limitantes superiores iguais ou muito próximos do valor da solução ótima; (iii) Christ/Fact produziu soluções em média $16,5 \%$ melhores que Wang, porém que demandaram um tempo computacional substancialmente superior (135 segundos em média). Além disso, Christ/ Fact produziu soluções melhores do que Christ nos exemplos $C W 3$ e $O F 2$.

Convém salientar que o método exato de CHRISTOFIDES \& HADJICONSTANTINOU (1995), baseado no algoritmo de otimização do subgradiente e num procedimento de busca em árvore, foi capaz de encontrar as soluções ótimas de todos os exemplos acima, no entanto os tempos computacionais foram bem elevados (em alguns casos chegaram à ordem de horas num microcomputador IBM 486). Ao compararmos Christ/Fact com outros métodos heurísticos da literatura, por exemplo, o método de FAYARD et al. (1998), este encontrou soluções inferiores às de Christ/Fact para os exemplos CW2 (2731), CW3 (1740) e $O F 1$ (2713), mas superior para o exemplo $O F 2$ (2586), com tempos computacionais da ordem de poucos segundos numa estação de trabalho Sparc-Server20.

\subsection{Exemplos gerados aleatoriamente}

Geramos, aleatoriamente, 20 exemplos com chapas de dimensões $(L, W)=(100,100)$ e $n=10$ peças, divididos em duas classes: exemplos com peças "grandes" e "pequenas". Essa classificação tem sido utilizada por alguns autores na literatura, como em BEASLEY (1985), MORABITO \& ARENALES (1995, 1996), e HIFI (1997).

- Exemplos com peças grandes: as dimensões $l_{i}, w_{i}, i=1,2, \ldots, n$, foram sorteadas (e em seguida arredondadas) de uma distribuição uniforme nos intervalos $[0,25 L, 0,75 L]$, $[0,25 \mathrm{~W}, 0,75 \mathrm{~W}]$, respectivamente.

- Exemplos com peças pequenas: as dimensões $l_{i}, w_{i}, i=1,2, \ldots, n$, foram sorteadas (e em seguida arredondadas) de uma distribuição uniforme nos intervalos $[0,20 L, 0,50 L]$, $[0,20 \mathrm{~W}, 0,50 \mathrm{~W}]$, respectivamente.
Em todos os exemplos, os valores de utilidade $v_{i}, i=1,2, \ldots, n$, foram considerados iguais às áreas $l_{i} w_{i}$ das peças, e as demandas $d_{i}$ foram sorteadas de uma distribuição uniforme no intervalo [1, 4]. Os demais dados destes 20 exemplos podem ser encontrados em SILVEIRA (1999). As Tabelas 2 e 3 apresentam os resultados obtidos para os exemplos com peças grandes e pequenas, respectivamente. Como estes exemplos foram gerados aleatoriamente, a solução ótima não é conhecida a priori. Portanto, só temos garantia de otimalidade quando a solução gerada em Christ ou Christ/Fact é factível e tem valor igual ao limitante superior (passos 3 ou 4 do algoritmo da seção 2.3). Nas tabelas, o símbolo "(1)" na coluna Ótima indica que o exemplo tem solução ótima desconhecida.

Como podemos observar, para os exemplos com peças grandes $(25 \%$ a $75 \%$ do tamanho da placa original), as soluções apresentadas em Christ e Christ/Fact são quase sempre ótimas e acompanhadas de um certificado de otimalidade; além disso, são superiores às soluções encontradas em Wang (em média mais de 15\%, conforme Tabela 2). Note que Christ/Fact supera Christ apenas no problema 5 (neste caso, não sabemos se a solução é ótima, mas sabemos que, caso não seja, o gap de otimalidade é, no máximo, 3,3\%), o que sugere que a heurística de factibilização é menos efetiva em problemas com peças grandes.

Para os exemplos com peças pequenas $(20 \%$ a $50 \%$ do tamanho da placa original), as soluções obtidas em Christ e Christ/Fact nem sempre são acompanhadas de um certificado de otimalidade (note que não sabemos se as soluções dos problemas $1,2,3,6,8$ e 10 na Tabela 3 são ótimas). Isso se deve ao fato de que agora cabem mais peças dentro da placa original (em comparação aos problemas com peças grandes), aumentando, assim, as chances de que o algoritmo de otimização de subgradiente gere soluções infactíveis (i.e., colocando mais peças no padrão de corte do que a demanda requerida). Contudo, as soluções obtidas em Christ/Fact ainda são melhores que as encontrados em Wang 
Tabela 2 - Resultados computacionais para 10 exemplos aleatórios com peças grandes, $\operatorname{com}(L, W)=(100,100)$, e $n=10$.

\begin{tabular}{|c|c|c|c|c|c|c|c|c|c|c|c|}
\hline \multirow{2}{*}{ Prob } & \multicolumn{2}{|c|}{ Dados } & \multicolumn{2}{|c|}{ Wang } & \multicolumn{3}{c|}{ Christ } & \multicolumn{2}{c|}{ Christ/Fact } & Ótima & Melh \\
\cline { 2 - 14 } & $|X|$ & $|Y|$ & Sol. & Tempo & Lim. & Sol. & Tempo & Sol. & Tempo & Sol. & $\%$ \\
\hline 1 & 24 & 30 & 8438 & $<1$ & 8828 & 8828 & 3 & 8828 & 3 & 8828 & 4,6 \\
\hline 2 & 26 & 13 & 6768 & $<1$ & 8244 & 8244 & $<1$ & 8244 & $<1$ & 8244 & 21,8 \\
\hline 3 & 35 & 24 & 6570 & $<1$ & 8180 & 8180 & 2 & 8180 & 2 & 8180 & 24,5 \\
\hline 4 & 33 & 22 & 8634 & $<1$ & 8799 & 8799 & 5 & 8799 & 5 & 8799 & 1,9 \\
\hline 5 & 32 & 37 & 8758 & $<1$ & 9507 & 8758 & 20 & 9194 & 16 & $(1)$ & 5,0 \\
\hline 6 & 40 & 25 & 7642 & $<1$ & 8464 & 8464 & 2 & 8464 & 2 & 8464 & 10,8 \\
\hline 7 & 27 & 27 & 6791 & $<1$ & 8751 & 8751 & $<1$ & 8751 & $<1$ & 8751 & 28,9 \\
\hline 8 & 31 & 35 & 9536 & $<1$ & 9715 & 9715 & $<1$ & 9715 & $<1$ & 9715 & 1,9 \\
\hline 9 & 40 & 26 & 5828 & $<1$ & 8919 & 8919 & $<1$ & 8919 & $<1$ & 8919 & 53,0 \\
\hline 10 & 35 & 31 & 8768 & $<1$ & 9611 & 8768 & 92 & 8768 & 92 & $(1)$ & 0,0 \\
\hline Média & & & $<1$ & & & 12 & & 12 & & 15,2 \\
\hline
\end{tabular}

(1) A solução ótima não é conhecida

Tabela 3 - Resultados computacionais para 10 exemplos aleatórios com peças pequenas, $\operatorname{com}(L, W)=(100,100)$ e $n=10$.

\begin{tabular}{|c|c|c|c|c|c|c|c|c|c|c|c|}
\hline \multirow{2}{*}{ Prob } & \multicolumn{2}{|c|}{ Dados } & \multicolumn{2}{|c|}{ Wang } & \multicolumn{3}{|c|}{ Christ } & \multicolumn{2}{|c|}{ Christ/Fact } & \multirow{2}{*}{$\frac{\text { Ótima }}{\text { Sol. }}$} & \multirow{2}{*}{$\frac{\text { Melh }}{\%}$} \\
\hline & $|X|$ & $|Y|$ & Sol. & Teтро & Lim. & Sol. & Tempo & Sol. & Teтро & & \\
\hline 1 & 51 & 43 & 9026 & $<1$ & 9446 & 9026 & 631 & 9238 & 621 & (1) & 2,3 \\
\hline 2 & 42 & 43 & 9022 & $<1$ & 9308 & 9022 & 68 & 9216 & 66 & (1) & 2,2 \\
\hline 3 & 45 & 41 & 8640 & $<1$ & 9356 & 8640 & 221 & 8932 & 137 & (1) & 3,4 \\
\hline 4 & 58 & 39 & 9518 & $<1$ & 9682 & 9682 & 1 & 9682 & 1 & 9682 & 1,7 \\
\hline 5 & 55 & 46 & 8649 & $<1$ & 9381 & 9381 & 3 & 9381 & 3 & 9381 & 8,5 \\
\hline 6 & 53 & 42 & 6568 & $<1$ & 9148 & 6568 & 367 & 8914 & 331 & (1) & 35,7 \\
\hline 7 & 34 & 50 & 8648 & $<1$ & 9656 & 9656 & $<1$ & 9656 & $<1$ & 9656 & 11,7 \\
\hline 8 & 50 & 49 & 9043 & $<1$ & 9891 & 9043 & 429 & 9533 & 427 & (1) & 5,4 \\
\hline 9 & 41 & 31 & 8238 & $<1$ & 8964 & 8964 & $<1$ & 8964 & $<1$ & 8964 & 8,8 \\
\hline 10 & 35 & 53 & 7958 & $<1$ & 9477 & 7958 & 188 & 7958 & 188 & (1) & 0,0 \\
\hline \multicolumn{3}{|l|}{ Média } & & $<1$ & & & 196 & & 178 & & 8,0 \\
\hline
\end{tabular}

(1) A solução ótima não é conhecida

(em média 8\%, conforme Tabela 3), apesar de os tempos computacionais serem bem maiores (178 segundos em média). Além disso, nos problemas onde a solução ótima não foi encontrada, Christ/Fact produziu soluções em geral melhores do que Christ (problemas 1, 2, 3, 6 e 8), indicando que a heurística de factibilização é mais efetiva em problemas com peças pequenas.

\section{Conclusões e Perspectivas para Pesquisa Futura}

Teste artigo estudamos o problema de corte bidimensional guilhotinado restrito. Para resolvê-lo, exploramos uma variação do método de CHRISTOFIDES \& HADJICONSTANTINOU (1995), baseada numa relaxação do espaço de 
estados de uma formulação de programação dinâmica do PGR (seção 2.2), num procedimento do tipo otimização do subgradiente (seção 2.3 ), e numa heurística de factibilização (seção 2.4). O desempenho computacional deste método heurístico foi avaliado resolvendo-se 6 exemplos da literatura (seção 3.1) e 20 exemplos aleatórios (seção 3.2), e comparando-se as soluções obtidas com as soluções da conhecida heurística de WANG (1983).

Os resultados mostraram que a abordagem foi capaz de produzir soluções melhores do que a heurística de Wang (com parâmetro $\beta=0,03$ ), com melhorias médias de $16,5 \%$ nos exemplos da literatura, e $15 \%$ e $8 \%$ nos exemplos aleatórios com peças grandes e pequenas, respectivamente. Além disso, muitas destas soluções são ótimas e, nos casos em que não são, a abordagem, partindo da solução ótima do problema irrestrito (relaxado), foi capaz de produzir um limitante superior relativamente próximo do valor da solução ótima do PGR, o que permite uma boa estimativa do gap de otimalidade (nos experimentos este limitante superior coincidiu com o valor da solução ótima em 5 dos 6 exemplos da literatura, em pelo menos 8 dos 10 exemplos aleatórios com peças grandes, e em pelo menos 4 dos 10 exemplos aleatórios com peças pequenas). Uma vantagem deste método é que, nos casos em que ele encontrou uma solução ótima, em geral também forneceu um certificado de otimalidade. Por outro lado, uma desvantagem dele foram os tempos computacionais, substancialmente superiores aos da heurística de Wang.

Apesar de a heurística de factibilização utilizada ser bastante simples, o presente método produziu resultados razoavelmente bons, se comparados aos de WANG (1983) e FAYARD et al. (1998), e parece ser mais efetivo nos exemplos com peças pequenas (Tabela 3 ) do que grandes (Tabela 2). Entretanto, o método, na sua versão atual, é superado pela abordagem em grafo-e/ou de MORABITO \& ARENALES (1996), capaz de produzir, para todos os exemplos aqui analisados, soluções no mínimo tão boas e com tempos computacionais bem inferiores. Outras heurísticas de factibilização mais elaboradas que a atual estão na nossa agenda de pesquisa para tentar melhorar o desempenho atual do método.

Outra perspectiva interessante para pesquisa futura seria um estudo aprofundado em uma fábrica de vidro ou placas de circuito impresso, aplicando a heurística de aspiração (seção 2) combinada com o método aqui proposto, para avaliar na prática o benefício desta abordagem com relação aos métodos utilizados pela empresa.

\section{Agradecimentos}

Os autores agradecem aos revisores anônimos pelos úteis comentários e sugestões. Esta pesquisa contou com apoio da FAPESP (processos 95/9522-0, 97/02508-7, 97/13930-1) e CNPq (processo 522973/95-7).

\section{Referências Bibliográficas}

ARENALES, M.; MORABITO, R.; YANASSE, H. Special issue: Cutting and packing problems. Pesquisa Operacional, v.19, n.2, p.107-299, 1999.

BEASLEY, J.E. Algorithms for unconstrained twodimensional guillotine cutting. Journal of the Operational Research Society, v.36, n.4, p.297-306, 1985.
BISCHOFF, E.; WAESCHER, G. Special Issue on Cutting and Packing Problems. European Journal of Operational Research, v.84, n.3, p.503-712, 1995.

CHRISTOFIDES, N.; HADJICONSTANTINOU, E. An Exact Algorithm for Orthogonal 2-D Cutting Problems Using Guillotine Cuts. European Journal of Operational Research, v.83, p.21-38, 1995. 
CHRISTOFIDES, N.; MINGOZZI, A.; TOTH, P. State-space relaxation procedure for the computation of bounds to routing problems. Networks, v.11, p.145-164, 1981.

CHRISTOFIDES, N.; WHITLOCK, C. An algorithm for two-dimensional cutting problems. Operations Research, v.25, n.1, p.30-44, 1977.

DOWSLAND, K.; DOWSLAND, W.B. Packing Problems. European Journal of Operational Research, v.56, p.2-14, 1992.

DYCKHOFF, H. Classification of Real World Trim Loss Problems. In: FANDEL, G. et al. Essays on Production Theory and Planning. Berlin: Springer-Verlag, 1992.

DYCKHOFF, H.; FINKE, U. Cutting and Packing in Production and Distribution: Typology and Bibliography. Heidelberg: Springer-Verlag, 1992.

DYCKHOFF, H.; SCHEITHAUER, G.; TERNO, J. Cutting and packing. In: AMICO, M.; MAFFIOLI, F.; MARTELLO, S. (eds.). Annotated bibliographies in combinatorial optimisation. New York: John Wiley \& Sons, 1997. p.393-414.

FAYARD, D.; HIFI, M.; ZISSIMOPOULOS, V. An efficient approach for large-scale twodimensional guillotine cutting stock problems. Journal of the Operational Research Society, v.49, p.1270-1277, 1998.

HERZ, J.C. Recursive computational procedure for two-dimensional cutting. IBM Journal of Research and Development, p.462-469, 1972.

HIFI, M. An improvement of Viswanathan and Bagchi's exact algorithm for constrained twodimensional cutting stock. Computers and Operations Research, v.24, n.8, p.727-736, 1997.

HINXMAN, A.I. The Trim Loss and Assortment Problems: A Survey. European Journal of Operational Research, v.5, p.8-18, 1980.

MORABITO, R.; ARENALES, M. Um Exame dos Problemas de Corte e Empacotamento. Pesquisa Operacional, v.12, n.1, p.1-20, 1992.
MORABITO, R.; ARENALES, M. Performance of Two Heuristics for Solving Large Scale TwoDimensional Guillotine Cutting Problems. INFOR, v.33, n.2, p.145-155, 1995.

MORABITO, R.; ARENALES, M. Staged and constrained two-dimensional guillotine cutting problems: An AND/OR-graph approach. European Journal of Operational Research, v.94, p.548-560, 1996.

MORNAR, V.; KHOSHNEVIS, B. A cutting stock procedure for printed circuit board production. Computers and Industrial Engineering, v.32, n.1, p.57-66, 1997.

MUKHACHEVA, E.A. Decision making under conditions of uncertainty: cutting-packing problems. The International Scientific Collection, Ufa, Russia, 1997.

OLIVEIRA, J.F.; FERREIRA, J.S. An improved version of Wang's algorithm for two-dimensional cutting problems. European Journal of Operational Research, v.44, p.256-266, 1990.

SILVEIRA, R.J. O Problema de Corte Bidimensional Guilhotinado e Restrito: Aplicação na Indústria de Circuitos Impressos. Dissertação (Mestrado) DEP, Universidade Federal de São Carlos, São Carlos, 1999.

SWEENEY, P.; PATERNOSTER, E. Cutting and Packing Problems: A Categorized, Applicationoriented Research Bibliography. Journal of the Operational Research Society, v.43, p.691-706, 1992.

VASKO, F.J. A computational improvement to Wang's two-dimensional cutting stock algorithm. Computers and Industrial Engineering, v.16, n.1, p.109-115, 1989.

VISWANATHAN, K.V.; BAGCHI, A. Best-first search methods for constrained two-dimensional cutting stock problems. Operations Research, v.41, n.4, p.768-776, 1993.

WANG, P.Y. Two Algorithms for Constrained TwoDimensional Cutting Stock Problems. Operations Research, v.31, p.573-586, 1983. 


\title{
A HEURISTIC METHOD BASED ON DYNAMIC PROGRAMMING FOR THE CONSTRAINED TWO-DIMENSIONAL GUILLOTINE CUTTING PROBLEM
}

\begin{abstract}
In this paper we study a particular case of two-dimensional cutting problems named constrained guillotine cutting (CGC). The CGC is an NP-hard problem that appears in different industrial processes of cutting rectangular plates, such as in the glass and circuit board industries. To solve the problem we present a variation of the exact method of CHRISTOFIDES \& HADJICONSTANTINOU (1995), based on a state space relaxation of a dynamic programming formulation of the CGC, a procedure of subgradient optimization type, and a feasibility heuristic. The result is a method without guarantee of optimality, however, faster and able to solve larger problems than the exact method of Christofides and Hadjiconstantinou. The computational performance of the approach is evaluated solving several examples of the literature as well as randomly generated examples, and comparing the solutions obtained with the ones of Christofides and Hadjiconstantinou's method and the well-known heuristic of WANG (1983).
\end{abstract}

Key words: cutting problem, constrained two-dimensional cutting patterns, dynamic programming, heuristics. 